\title{
Descriptors for Electron and Hole Charge Carriers in Metal Oxides
}

Daniel W. Davies, ${ }^{*, \dagger, \|}$ Christopher N. Savory, ${ }^{\ddagger} \|$ Jarvist M. Frost, $₫$ David O. Scanlon, ${ }^{\ddagger}, \|$ Benjamin J. Morgan,,,$\|$ and Aron Walsh*,,,$\|$

$\dagger$ Department of Materials, Imperial College London, London SW7 2AZ, United Kingdom

$\ddagger$ Department of Chemistry, University College London, 20 Gordon Street, London WC1H $0 A J$, United Kingdom

9Department of Physics, Imperial College London, London SW7 2AZ, UK

$\S$ Department of Chemistry, University of Bath, Claverton Down, Bath BA2 7AY, United

Kingdom

\|The Faraday Institution, Quad One, Harwell Science and Innovation Campus, Didcot, UK

$\perp$ Diamond Light Source Ltd., Diamond House, Harwell Science and Innovation Campus, Didcot, Oxfordshire OX11 ODE, United Kingdom

\#Department of Materials Science and Engineering, Yonsei University, Seoul 03722, Korea

E-mail: d.davies16@imperial.ac.uk; a.walsh@imperial.ac.uk

\section{Supplementary Information}


Electron $\mathrm{E}_{\text {polaron }}(\mathrm{meV})$

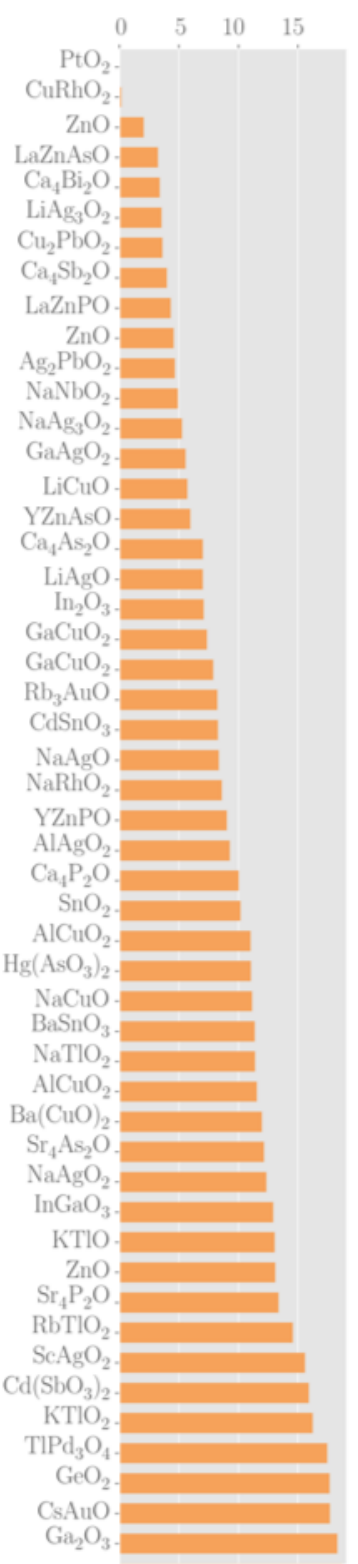

$\begin{array}{lllll}0 & 10 & 20 & 30 & 40\end{array}$

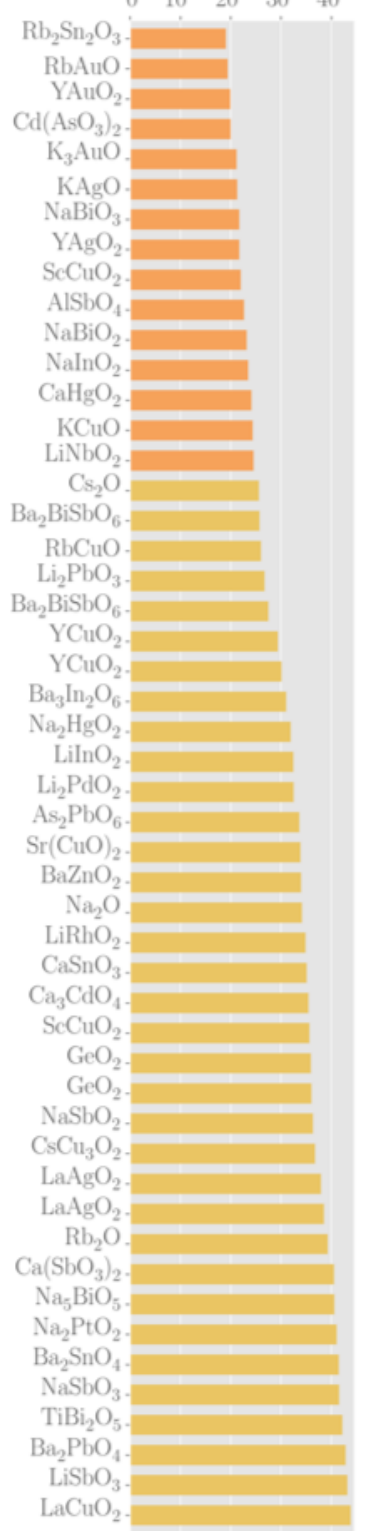

Hole $\mathrm{E}_{\text {polaron }}(\mathrm{meV})$

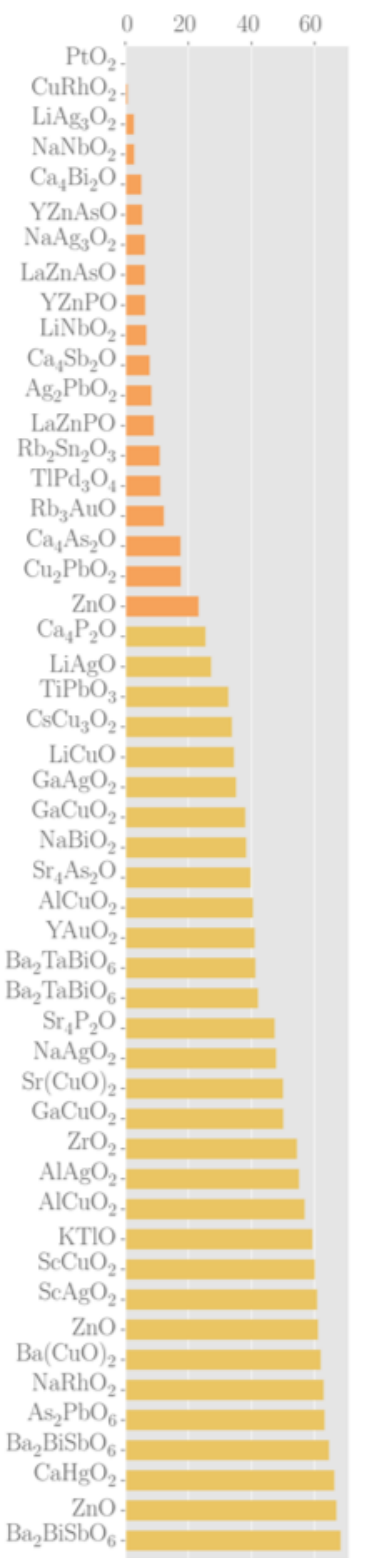

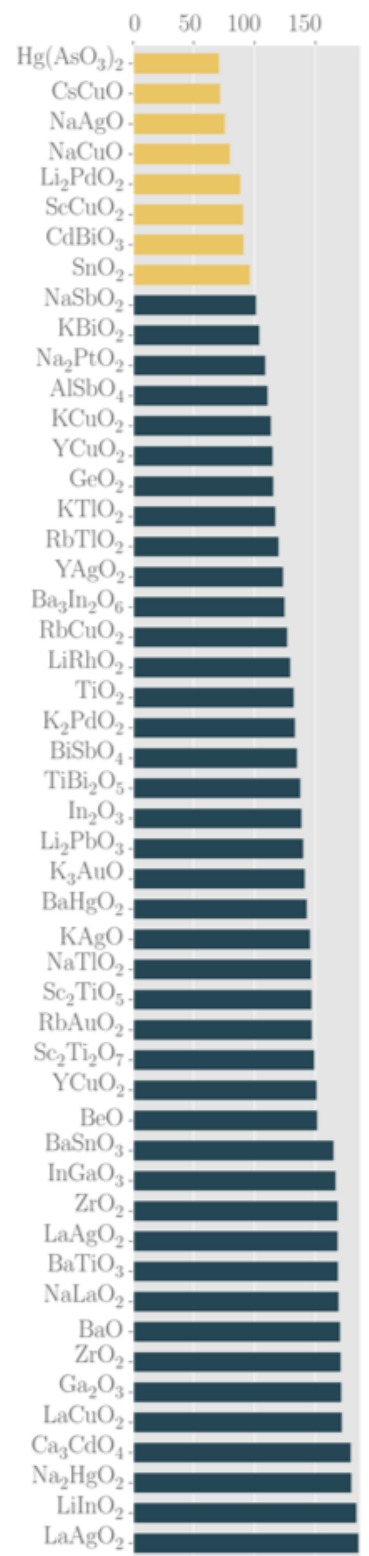

Figure S1: Electron and hole polaron binding energies calculated using the effective masses and dielectric constants from public data-sets. Some compositions have multiple entries due to polymorphism. 

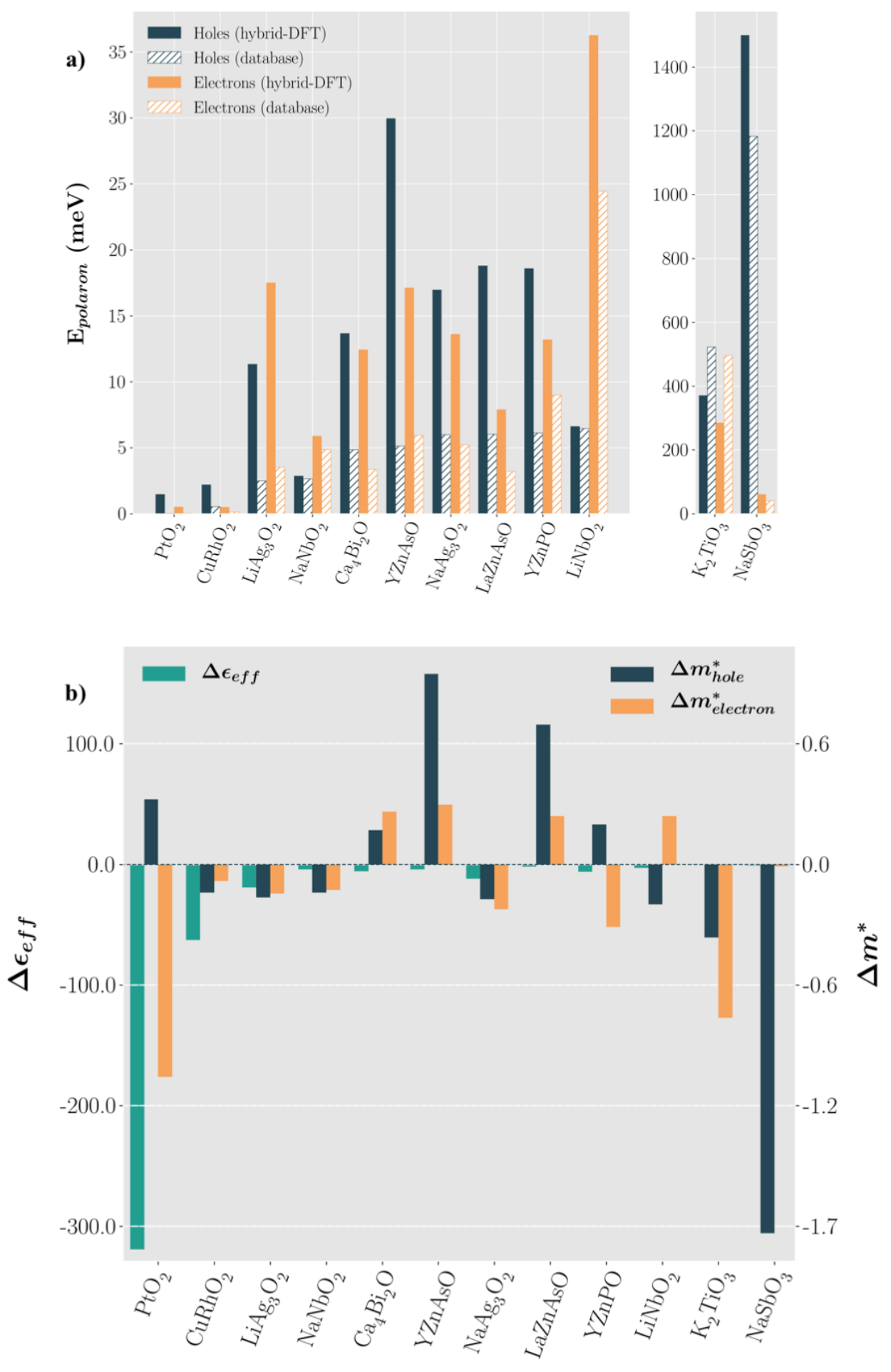

Figure S2: a) Polaron energy ( $\mathrm{E}_{\text {polaron }}$ ) values as calculated using hybrid-DFT (solid bars) and database (hatched bars) for electrons (orange bars) and holes (blue bars). b) Absolute difference (database derived minus Hybrid-DFT derived) in $\epsilon_{\text {eff }}$ (green bars) and effective mass $\left(m^{*}\right)$ for electrons (orange bars) and holes (blue bars). 


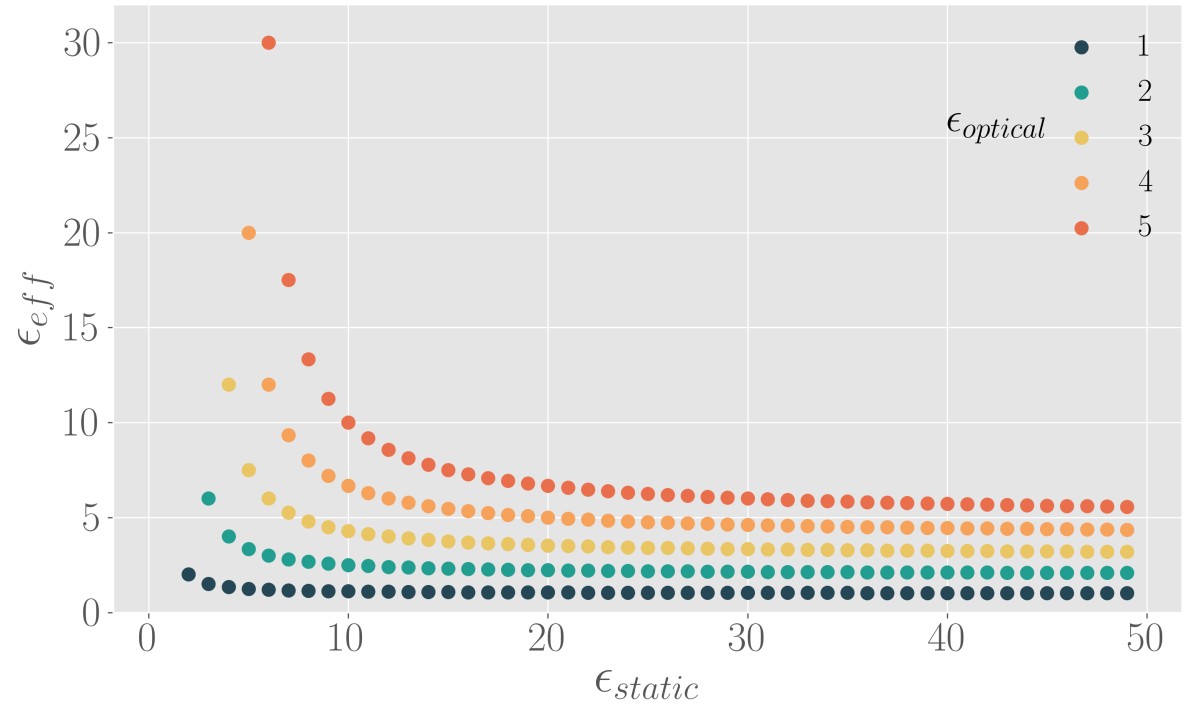

Figure S3: Visualization of the variation of $\epsilon_{\text {eff }}$ (y-axis) with $\epsilon_{s}$ (x-axis) at different fixed values of $\epsilon_{\infty}$. For $\epsilon_{s}>20$ the system is in a strong screening regime where $\epsilon_{\text {eff }}$ shows little variation. 